\title{
Relaciones públicas digitales. Análisis de las estrategias de comunicación de los think tanks
}

\section{Digital public relations. Analysis of think tank communication strategies}
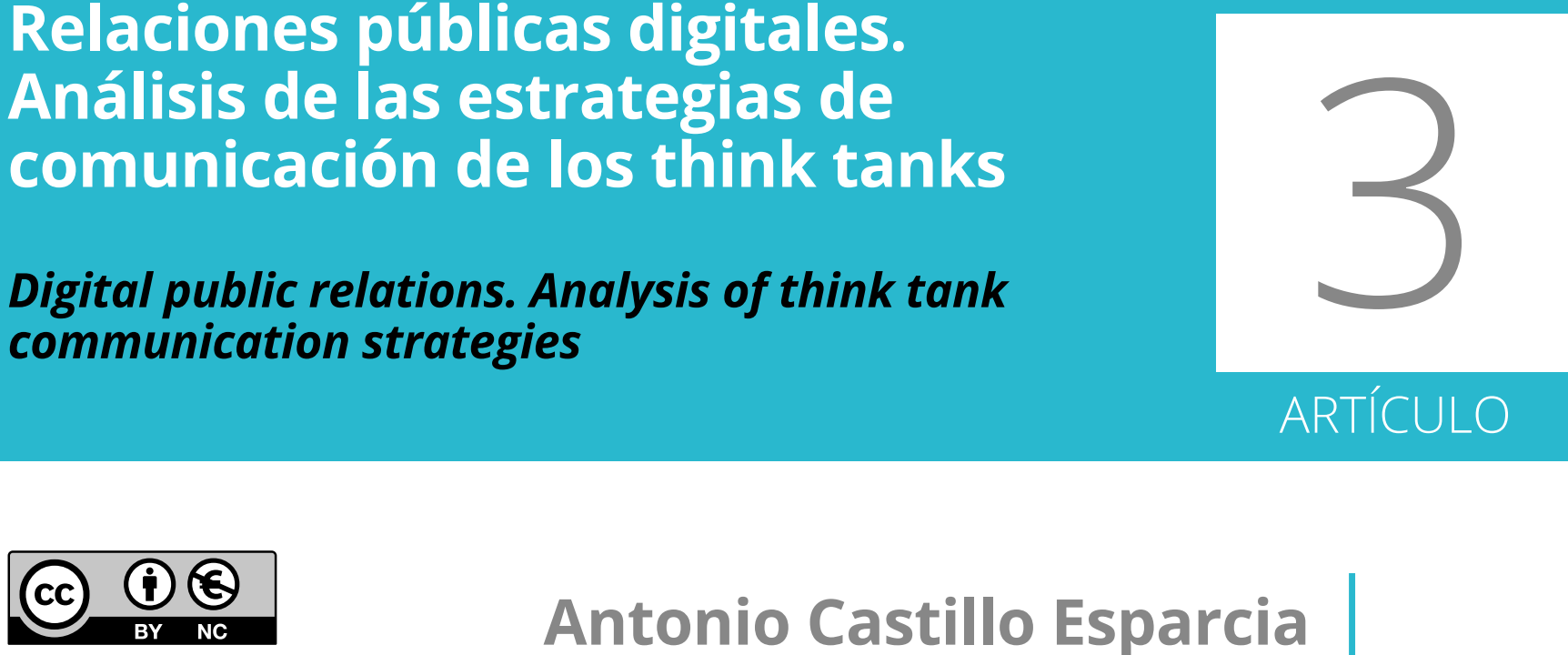

\section{Antonio Castillo Esparcia}

Facultad de Ciencias de Comunicación. Universidad de Málaga

Director del Departamento de Comunicación Audiovisual y Publicidad. Profesor de Publicidad y Relaciones Públicas 2.0.

acastilloe@uma.es

orcid.org/0000-0002-9751-8628

\section{Emilia Smolak Lozano}

Facultad de Ciencias de Comunicación. Universidad de Málaga

Profesora de Comunicación Audiovisual, Publicidad y Relaciones Públicas en Universidad de Málaga. Autora de varias publicaciones sobre comunicación digital.

esmolaklozano@uma.es

orcid.org/0000-0001-8193-8786

Fecha de recepción: 03 de marzo de 2016 / Aceptación: 29 de mayo de 2017

\section{Resumen}

El objetivo es investigar el uso de relaciones públicas 2.0 en la página web de los mejores 40 think tanks asiáticos (Go To Global Think Tanks Report, 2013), utilizando como base de análisis la información aportada por los sitios web de los think tanks analizados. De este modo, mediante un análisis cuantitativo del contenido a partir de una ficha de análisis elaborada al tal propósito hemos estudiado que tipo de información publican los think tank asiáticos en sus páginas web en su comunicación como aspecto clave de las relaciones públicas 2.0.

\section{PALABRAS CLAVE}

Relaciones públicas 2.0, comunicación 2.0, think tanks, web social 


\section{Abstract}

The objective of this paper is the study of the use of public relations 2.0 by Asian think tanks on their websites, using the information provided there as a basis for analysis. The sample includes the 40 top-ranked global Asian think tanks in the annual Global Go To Think Tank Index Report drawn up by McGann in 2015. Through quantitative content analysis of a specially designed database, the information on these Asian think tank websites was studied in terms of public relations 2.0 content.

\section{KEYWORDS}

Public relations 2.0. communication 2.0, think tank, social web

\section{INTRODUCCIÓN}

Las nuevas tecnologías han sido bien aceptadas tanto a nivel de uso individual como a nivel de organización gracias a los beneficios de la comunicación interactiva, dialógica y a la capacidad de conectar con la gente (Valentini, 2015: 170), lo que fomenta nuestra conectividad de forma omnipresente (Van Dijck, 1991). De este modo, la comunicación a través de las nuevas tecnologías se vuelve más transparente, tal como indica García Cuenca, citado por Aced, Arqués y Benitéz (2009).

Wilcox, Cameron y Xifra (2012: 54-60) destacan el desarrollo tecnológico, el papel dominante de las nuevas tecnologías de comunicación, el papel estratégico, la importancia de la relación y la percepción (gestionar las relaciones) como los elementos que caracterizan la fase de madurez en el desarrollo histórico de relaciones públicas hacia el modelo objeto de nuestro estudio, como es el espacio 2.0. Estos elementos implican en una repercusión visible e impactante en las estrategias y herramientas desempeñadas en estos modernos espacios de comunicación.

Las Relaciones Públicas 2.0 se desarrollan a partir de la adopción de las herramientas tecnológicas de comunicación: Internet y más recientemente los Social Media. De esta forma, facilitan el diálogo y la conversación entre la organización y el público a base de la información que facilitan en estos nuevos sitios online. Dentro de las TIC que fueron los pioneros para las estrategias online de Relaciones Públicas encontramos las páginas web (Holtz, 2002). Se han visto influidas por el cambio desde el paradigma 1.0 que se basaba en la información distribuida de forma unidireccional hasta el paradigma 2.0 cuyo modo operativo abarca el uso de las herramientas que facilitan un contacto interactivo (Liberos et al, 2013; Capriotti, Carretón y Castillo-Esparcia, 2016).

El desarrollo de comunicación a través de las herramientas tecnológicas ha sido un reto para las estrategias comunicativas de los think tanks (Selee, 2013; McGann, 2007) que se han visto obligadas a adoptar las soluciones online a sus tácticas tales como son la difusión del contenido, comunicación de información, influencia y visibilidad de la organización. Sobre todo, este asunto ha cobrado importancia en las regiones en desarrollo como el continente asiático donde se observan los think tanks globales ante de los retos de una comunicación globalizada que residen en los países en vía de desarrollo, por un lado, y en las democracias y economías avanzadas, por otro.

El objeto de este estudio es la comunicación externa a través de las políticas de relaciones públicas 2.0 de los think tanks más importantes a nivel global, especialmente en las regiones en 
los procesos de transformación: el continente asiático que ha experimentado en las últimas décadas el cambio más significativo (Krastev, 2001; Stone, 2005). En los países de Asia y el Pacifico, excluyendo a Japón, India, Corea del Sur o China ${ }^{1}$ que cuentan con el mayor número de think tanks, los países con el mayor número de los think tanks es Australia (63), Taiwán (52), Bangladesh (35) y, Hong Kong (30) e Indonesia (27). Adicionalmente, la mayor parte de los estudios se centran en los think tanks norteamericanos (Abelson, 2001 y 2006) o estadounidenses (Xifra, 2008), y no en las regiones emergentes, mientras que la región del Sureste de Asia y Pacifico, no tienen el mismo nivel de representación en los estudios a pesar de ser las regiones con el mayor número de think tanks sólo por detrás del continente americano.

El objetivo es construir el perfil del uso de relaciones públicas 2.0 en la web de los think tanks asiáticos utilizando como base de análisis la información aportada por los sitios web de los think tanks analizados.

\section{MARCO TEÓRICO}

\subsection{DEFINICIÓN DE THINK TANK.}

A pesar de una tradición científica de más de medio siglo sobre el fenómeno de los think tanks parece que esta cita sigue siendo la que refleja con mayor fidelidad el estado del discurso científico acerca de este tipo de organizaciones. Para poder definir este fenómeno, es necesario saber que a pesar de un campo limitado (Rich, 2004) de los estudios sobre los think tanks es importante destacar las dos grandes tradiciones que lo dominan (Krastev, 2001).
Por un lado, nos encontramos con los estudios institucionales representados por Weaver (1989), McGann y Weaver (2000) o Smith (1991), que investigan la creación, formas organizacionales, criterios de distinción, modelos de gestión y aspectos relacionados con dicha gestión. Por otro lado, contamos con la corriente de investigación guiada por una pregunta fundamental: "¿cómo las ideas influyen las políticas?" (Hall, 1990) con un enfoque especial en la función social de utilizar el conocimiento y no tanto en sus características institucionales (Krastev, 2001; Abelson, 2006). Los representantes de este flujo intentan determinar cómo las estrategias comunicativas hacen las ideas poderosas (Krastev, 1999: 18).

Teniendo presente la multitud de conceptos por los cuales se definen los think tanks, los autores nos proponen una serie de criterios que nos puedan ayudar a distinguir los think tanks, desde un punto de vista institucional que comprende una estructura propia, una actividad con la intención de permanencia, distintos modelos de organización, labor de experto y orientación sin ánimo de lucro.

Los think tanks no sólo se ven influidos por los cambios de carácter político, económico o social en los contextos en las cuales se establecen y operan sino, también, por los procesos globales que les afectan provenientes del mundo tecnológico, trayendo las nuevas herramientas de comunicación. Teniendo en cuenta que la comunicación de los think tanks es una de las actividades principales que engloba, fundamenta y facilita la realización de los demás funciones y roles políticos de los think tanks en relación con su entorno externo, relaciones públicas resultan una herramienta básica y principal en su ámbito de actuación. 
Sin embargo, el desarrollo de las nuevas tecnologías de comunicación crea nuevos retos y oportunidades para los think tanks: "Digitalization has largely changed the power relations" (Van Ruler, 2015: 187-188). A continuación, el autor alega que la digitalización de la sociedad y los comportamientos de los públicos digitales dan lugar a cambios sustanciales del entorno comunicativo de las organizaciones, cambiando las herramientas de comunicación y de relaciones públicas (Zerfass, Tench, Verčič, Verhoeven y Moreno, 2014). Observamos un desarrollo intenso de este fenómeno a partir de los años noventa y del boom de Internet que tanto ha afectado al funcionamiento de la sociedad contemporánea (Slevin, 2009).

Partimos de la premisa de que la comunicación mediante la página web como la herramienta de relaciones públicas 2.0 más destacada (Holtz, 2002; Liberos, et al. 2013; Aced, 2013), es uno de los factores claves de éxito de los think tanks en cuanto a sus actividades en formulación de las políticas en estas regiones en el siglo XXl.

\subsection{RELACIONES PÚBLICAS 2.0.}

Dada la importancia de una planificación y gestión adecuada de la comunicación política 2.0 de los think tanks en base a relaciones públicas políticas en este sector, es conveniente en primer lugar referirse a las consideraciones de Barquero y Pérez (2010: 117-121), que destacan la importancia de relaciones públicas para las relaciones internacionales modernas y como agente de la transformación social al respecto de su poder, no sólo de transmitir la información sino de cambiar e impactar los entornos (público y opinión pública): "relaciones públicas representan hoy un nuevo instrumento de integración y desarrollo (..) en los tiempos de cambio".
Las tendencias actuales hablan de gestión de relaciones, diálogo, interés del público, fondo multicultural, transparencia, ampliación del papel de relaciones públicas, énfasis en la evaluación, gestión de ciclo de noticias 24/7 y crecimiento constante de los medios digitales que requiere una adaptación y aprendizaje continuos. Seitel (2002) considera relaciones públicas online como el instrumento de diferenciación en la red. En definitiva, una de las bases que ayudan a entender relaciones públicas 2.0 es la afirmación de Rojas Orduña (2012) sobre la capacidad de relaciones públicas de crear contactos y conversaciones entre diferentes stakeholders de forma neutral, respetuosa y marcando las fronteras de sus distintas especialidades. Solis y Breakenridge (2010), observan la transformación de relaciones con los públicos en relaciones públicas 2.0.

Partiendo de las aportaciones de Duhé (2015) y Verčič, Verčič y Sriramesh (2015), así como basándonos en los análisis de varios investigadores de relaciones públicas offline y online a lo largo del desarrollo de este ámbito académico, podemos distinguir cuatro elementos esenciales que determinan la definición de relaciones públicas 2.0:

- Relación-interacción.

- Públicos formados dentro las audiencias digitales.

- Conversación en forma del diálogo.

- e-influencia.

Su concepto básico radica en la participación y contribución con contenido, información y consejos, construyendo un portfolio online del conocimiento que realce la reputación de la organización, estimule la marca y aumente la presencia del perfil del negocio con la finalidad de alentar la lealtad del público. Se trata de ganarse la confianza, lo que al fin y al cabo 
no tiene precio. La página web fue destacada como la herramienta principal para cumplir tal fin (Capriotti et al., 2016; Aced, 2013; Liberos et al., 2013, Rojas Orduña, 2012).

La página web interactiva según la metodología de Liberos (et al.,2013: 220-227) y Aced (2013) es la tarjeta de presentación de la organización. Los estudios desde 1992 hasta 2009 sobre la aplicación de páginas web en relaciones públicas resaltan su dimensión interactiva como elemento crucial para establecer las relaciones (Kent, Taylor y White, 2003; Kent y Taylor, 1998) y confirman la importancia del diseño, públicos, estrategias de comunicación diferenciadas, perspectiva internacional y herramientas dialógicas (Duhé, 2015). Aced (2013) la denomina escaparate digital donde ejerce el control sobre los contenidos. Debe ser un sitio dinámico que permite cambios y modificaciones. Una página web tiene que contextualizar sus contenidos y definir bien sus audiencias (Rojas Orduña, 2012). Debe ser interactiva para fomentar la interactividad y conectividad con los usuarios e integrada con los Social Media para facilitar la expansión y difusión de sus contenidos. Su objetivo es proyectar la identidad e imagen deseada y contribuir a gestionar las impresiones y percepciones de los públicos objetivos. Su objetivo general y primordial es establecer el contacto y relación con los stakeholder. Resumiendo, los autores anteriormente mencionados indican una serie de elementos que la página web debería poseer para cumplir con los requisitos de relaciones públicas 2.0:

- Filosofía Pull por encima de Push.

- Accesible.

- Buen posicionamiento y visibilidad en buscadores.

- Interactividad: herramientas interactivas para establecer el diálogo con los stakeholders; para ello pueden servir las redes sociales integradas en la pá gina web.

- Integración con las redes sociales.

- Navegación efectiva.

- Experiencia satisfactoria por parte del usuario.

- Comunicación visual: facilidad de uso, confianza y credibilidad.

- Contenidos útiles y de valor.

- Agilidad y rapidez.

- Organización, estructura y navegabilidad.

- Personalización.

- Usabilidad y navegabilidad.

- Diseño.

- A esto hay que añadir optimización móvil, presentación visual atractiva de los contenidos, lenguaje (claridad, comprensión, formal/ informal), idiomas.

La página web de una organización realiza diversos objetivos de relaciones públicas: ofrecer de forma disponible y accesible la información corporativa y organizacional, atraer y fidelizar nuevos colectivos, establecer y acelerar el diálogo con los stakeholders, mantener relaciones con los medios de comunicación, mejorar el conocimiento sobre la organización y su marca, apoyar la imagen con información positiva, promover sus contenidos e ideas, así como incrementar la satisfacción y experiencias positivas (Rojas Orduña, 2012:248).

De este modo la página web debe contener los siguientes contenidos: información organizacional (misión, visión, valores, áreas de actuación, historia, plantilla y organigrama), información sobre sus productos o servicios, información financiera, información sobre el 
compromiso con la comunidad, sala de prensa virtual, contacto con los recursos humanos, datos de contacto y política de protección de datos (Rojas Orduña, 2012:248).

\section{METODOLOGÍA.}

De este modo, mediante un análisis cuantitativo del contenido a partir de una ficha de análisis elaborada al tal propósito hemos estudiado que tipo de información publican los think tank asiáticos en sus páginas web como la herramienta principal de sus estrategias de relaciones públicas en el mundo digitalizado: misión, visión y valores, la temática y área de especialización, así como el ámbito de actuación que se pretende a través de sus actividades comunicativas. Adicionalmente, con el objetivo de comprobar el grado de transparencia en su comunicación como aspecto clave de las relaciones públicas 2.0, hemos investigado si está disponible la información sobre el tamaño y la estructura del empleo, año de creación, estatus y pertenencia a los organismos estatales e internacionales, y por último la información sobre las finanzas en su fundación, financiación de su actividad e informes financieros. Como último, vamos a examinar el uso de las herramientas interactivas para determinar el grado de traspaso hacia la web.2.0.

La muestra comprende los 40 think tanks asiáticos globales indicados como los mejores por el estudio anual de McGann -Go To Global Think Tanks Report del año 2013. La metodología se basa en el análisis de contenido a través de una ficha de análisis creada especialmente para tal fin. Los variables recogidos en el estudio son dicotómicos (SI/NO), cualitativos y continuos y son los siguientes:
- Presencia a través de su página propia

- país de procedencia,

- año de creación,

- estructura de plantilla compuesta por 2 variables: número de empleados de dicados a investigación, número de empleados administrativos

- tamaño de la organización contada en el número total de empleados

- ámbito de actuación

- temas de especialización

- el estatus independiente o dependiente de la organización

- la pertenencia o no las redes o think tanks globales

- fuentes de financiación en el momento de creación

- fuente de ingresos

- grado de transparencia en revelar la información financiera: falta de información, información sobre los fondos iniciales a la hora de crear la organización e información completa.

El proceso de recopilación de datos tuvo lugar entre el 20 y el 30 de junio de 2016 para asegurar la información actualizada. Las unidades de análisis eran las páginas web corporativas de las organizaciones que forman parte de la muestra con sus apartados dedicados a la organización: los apartados Sobre Nosotros (About Us) y Plantilla (Staff). En el paso siguiente, los variables han sido codificados y los datos fueron tratados estadísticamente en el programa de análisis estadístico. 


\section{LA PRESENCIA DE LOS THINK TANKS ASIÁTICOS EN SUS PÁGINAS WEB.}

\subsection{DATOS GENERALES.}

En primer lugar, hemos investigado el hecho de tener o no tener una página web como herramienta básica de comunicación corporativa en el mundo online.

\section{Web corporativa}

$\square$ Independet web $\square$ No independet web $\square$ no web

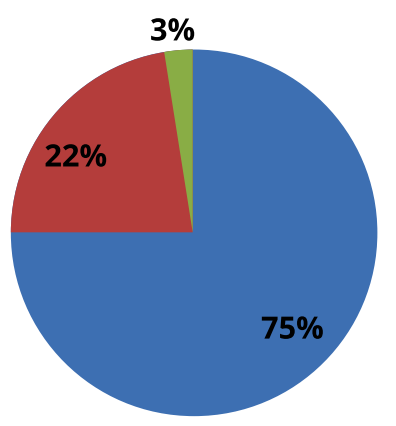

Figura 1. Presencia en la web. Fuente: elaboración propia.

El 75\% posee su propia web corporativa de forma independiente de otros organismos, mientras que un $22 \%$ tiene su web corporativa o su información corporativa incluida en la página web de otros organismos. Por otro lado, también encontramos que un think tank (BDIPSS de Brunei) no tiene página web.

A lo largo del estudio hemos descubierto que las páginas tienen un diseño anticuado, no son optimizadas para el móvil, experimentan problemas de carga y técnicos de visualización o accesibilidad y están saturadas de información con una estructura muy confusa en la mayoría de casos. Esta dimensión es importante para el estudio ya que la información sobre las organizaciones investigadas procede de sus páginas web.
Los think tanks sin una página web propia son los siguientes y están relacionados con la estructura organizativa de las instituciones a que pertenecen: IWEP de Vietnam, ISDS de Filipinas, SDSC de Australia, IDSS de Singapur, CSS de Nueva Zelanda y EAl de Singapur.

Como se puede comprobar, la mayoría de los think tanks investigados han adaptado las soluciones TIC a su comunicación y las han utilizado dentro de su estrategia de Relaciones públicas ya que la información facilitada sobre la organización cumple con los mínimos requisitos de aplicación profesional de relaciones públicas. De este modo, la información corporativa ofrecida a estas páginas, en todos los casos de los think tanks, con la presencia en la página web, ya sea propia o no, demuestra al menos una descripción corta de la organización: su desarrollo, actividades y funciones, objetivos, etc. En la mayoría de los casos son textos muy concisos y en muchas ocasiones deberían ser más desarrollados en cuanto a la información disponible.

En resumen, alrededor de $85 \%$ de los think tanks asiáticos dispone en su página web de la información sobre misión y visión, pero sólo algunos casos, incluyen los valores adicionalmente. El $80 \%$ de las organizaciones de esta región además facilita las biografías detalladas de sus investigadores, así como presidentes, consejos delegados o ejecutivos, pero en la mayoría de los casos no se facilita el contacto a estas personas. Sin embargo, todas las páginas demuestran la información detallada de su ubicación, modos de llegar y contacto. Cabe destacar, que el correo-electrónico no es la forma de contacto presentada con mayor frecuencia, más bien al contrario, este tipo de contacto está muy protegido. Asimismo, podemos constatar que la información sobre la organización está incluida en la página web y forma parte de 


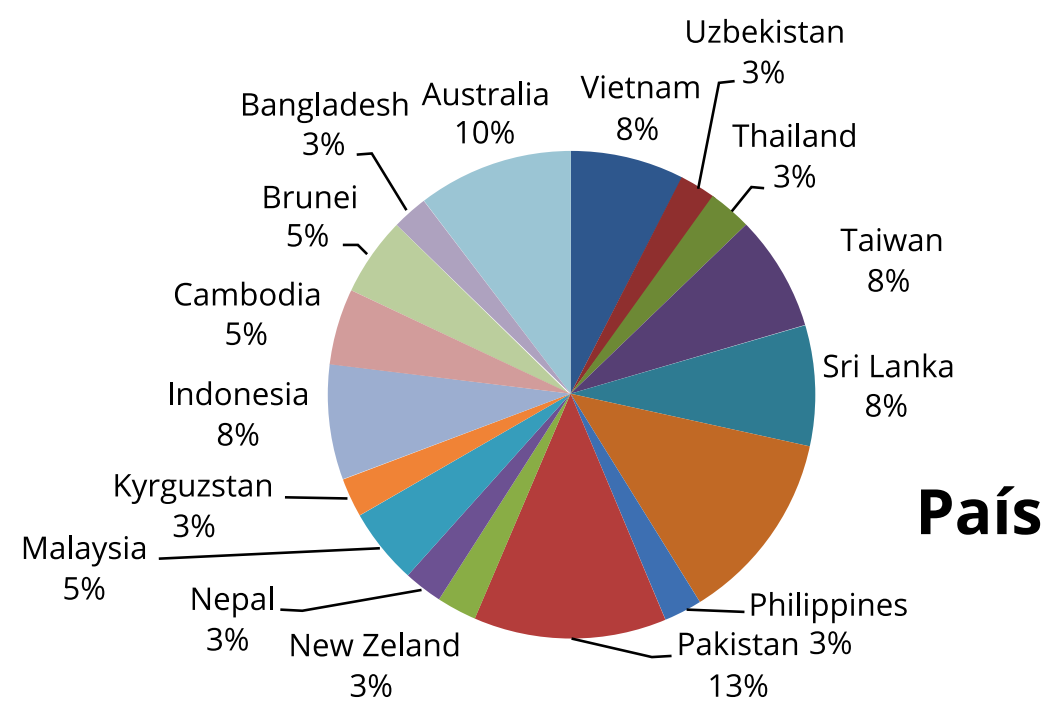

Figura 2. País de procedencia. Fuente: elaboración propia.

las estrategias de relaciones públicas de estas organizaciones.

En segundo lugar, nos centramos en el país de procedencia y el año de creación. (Figura 2)

Como demuestra la figura 2, los tres países: Singapur, Pakistán y Australia representan el 36\% del total de los think tanks investigados en cuanto a su procedencia. El mayor número de los think tanks recogidos en el índice del top think tanks de Asía y Pacífico que constituye la muestra procede de estos tres países: 5 de Singapur, 5 de Pakistán y 4 de Australia. Sri Lanka, Indonesia, Taiwán y Vietnam representan cada uno el $8 \%$ del total de los think tanks de esta región. En este sentido, podemos concluir que estos países destacan por la excelencia de sus think tanks, tal como lo recoge el índice Go To Think Tanks, así como en la referencia que otorgan a estos organismos en sus procesos democráticos. Adicionalmente, cabe destacar la representación de los países en vías de desarrollo y de las partes centrales o fronterizas del continente asiático, tales como: Kirguistán, Uzbekistán, Bangladesh y Cambodia, lo que demuestra el crecimiento de la sociedad de- mocrática y cívica en estas zonas y la consolidación de reglas de la comunicación política. Además, es importante ver la variedad geográfica de procedencia de los think tanks de esta región que no se limita a los países con la posición fuerte en la escena política.

La diversidad de procedencia en cuanto a los países también se refleja en caso de la antigüedad de las organizaciones en cuestión, lo que demuestra la Figura 3.

El desarrollo de las organizaciones analizadas abarca un periodo de casi un centenario, empezando con la más antigua Australian Institute of International Affairs (AllA) establecida en 1924 y terminando con Institute of Defense and Strategic Studies (IDSS) de Singapur, fundada recientemente en 2012.

El desarrollo demuestra dos periodos muy importantes de la historia de los think tanks en esta región. Primero, el boom de creación de los think tanks en las décadas de 1960 y 1970 (11 en total) e incluso entrando en la década de los 80' (5 en total), así como a partir de nuevo siglo (2000-2012), cuando se crearon 11 think tanks. El segundo boom refleja, por un lado, 
Año

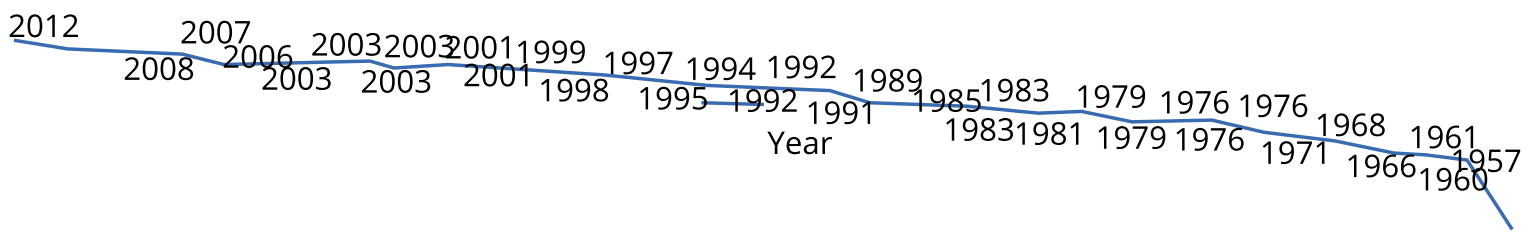

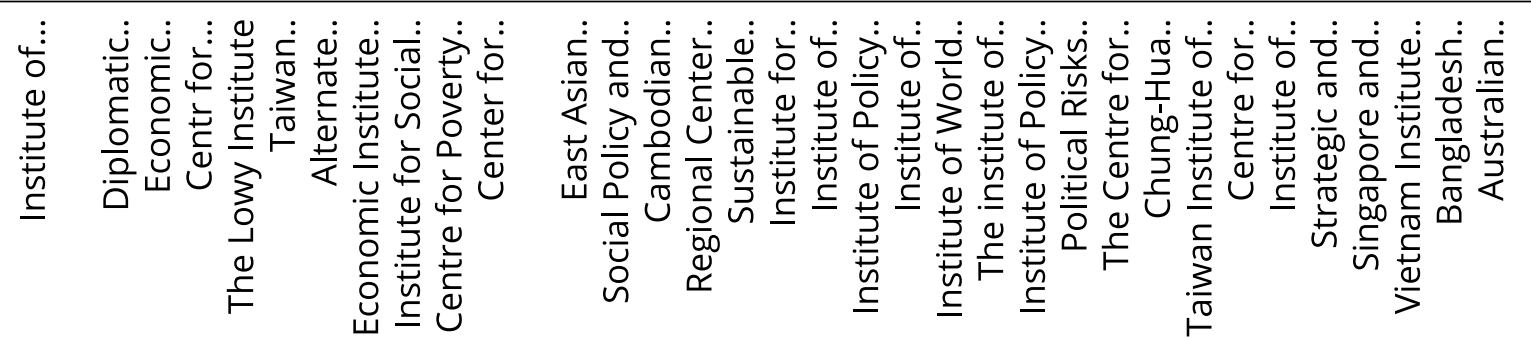

Figura 3. El año de creación de los think tanks. Fuente: elaboración propia.

los procesos de avances democráticos en esta aparte del mundo, así como el creciente significado de este tipo de organizaciones en los procesos políticos y democráticos en estos países que se traduce en el aumento de este tipo de organizaciones en el panorama público asiático y el debate democrático.

\subsection{AREAS DE ACTIVIDAD.}

Esta importancia creciente de los think tanks a lo largo del último siglo en Asia también se refleja en los temas tratados por este tipo de organizaciones.

Según los datos aportados en sus páginas web por los propios think tanks, la mayoría de ellos se especializa en la temática internacional de las relaciones internacionales y políticas extranjeras (international affairs y foreign policy), así como las cuestiones de desarrollo en sus dimensiones socio-económicas (development issues) en sus países de origen y región, y por último las cuestiones de defensa y seguridad militar. Además, encontramos los think tanks especializados en temas de desarrollo como ISET de Nepal o en temas de paz y conflictos, como lo demuestra el caso de FTl de Kirguistán. La diversidad de cuestiones socio-económicas que abarcan educación, salud, emprendimiento, pobreza, igualdad, entre otras, demuestra la complejidad de la realidad en la que operan los think tanks asiáticos y la importancia de su rol en enfrentar estas cuestiones en los distintos países. La seguridad y las relaciones internacionales permanecen en las que tienen mayor peso para los poderes políticos en la gran mayoría de los países investigados. Democracia junto con la investigación de las políticas públicas es otra de las cuestiones que marca la agenda política de los países en vías de desarrollo. Sólo un think tanks, ISET de Nepal, se ocupa de temas medioambientales mientras que CEPA de Sri Lanka se centra en una cuestión de forma estratégica: la pobreza.

\subsection{EL MODELO ORGANIZACIONAL.}

El promedio de empleados en los think tanks de la región de Asia y Pacífico es de 18 empleados, indicando claramente que en la mayoría de los casos contamos con las organizaciones 


\begin{tabular}{|c|c|c|c|c|c|}
\hline & Investigadores & Administración & $\begin{array}{l}\text { Número de } \\
\text { empleados }\end{array}$ & $\%$ Research & $\%$ Admin \\
\hline Promedio: & 14 & 9 & 18 & $40 \%$ & $26 \%$ \\
\hline
\end{tabular}

Figura 4. Tamaño de plantillas y estructura de empleo. Fuente: elaboración propia.

pequeñas o medianas. En cuanto a la estructura de los empleados, en general la mayoría de la plantilla la constituyen los investigadores (40\%), con un promedio de 14 investigadores por organización (Figura 4).

La administración supone el menor porcentaje de la plantilla con un promedio del $26 \%$ y 9 empleados por organización. A continuación, hemos destacado los think tanks y su estructura de organigrama (Figura 5).

Los think tanks donde la investigación representa el mayor peso en cuanto a la actividad principal reflejada por la estructura de empleo son: EIC, IDSS, ISIS de Tailandia y Malaysia, ISEAS, CSIS, The Lowy Institute y EAl, representando los países como Indonesia, Sri Lanka, Australia, Singapur y Cambodia. Los think tanks de Brunei, Pakistán, Bangladesh, Sri Lanka o Vietnam son donde las plantillas de investigadores constituyen el 50\% o hasta el 56\%, alcanzando cierto equilibrio de empleo entre administración e investigación. Sin embargo, tener la mayor plantilla involucrada en la investigación (más del 60\%) es la característica más común para los países desarrollados como Australia, Singapur, Malaysia o Nueva Zelanda. Por otro lado, podemos observar los casos en las cuales la administración forma la mayor parte de la plantilla, en ejemplos tales como: CICP de Cambodia, SIIA de Singapur o AllA de Australia o los think tanks de Vietnam y Taiwán. En este caso, la investigación puede venir de los acuerdos de colaboración que no supone el coste para la organización, sin embargo, esta cuestión requiere una investigación más profunda que no es objeto de este estudio. El caso único es FTI de Kirguistán donde dentro de la plantilla no se contratan los investigadores.

A continuación, podemos destacar los think tanks más grandes y con el mayor número de los investigadores y administración donde se puede observar que FTI de Kirguistán, VIE de Vietnam y CICP de Cambodia son los think tanks con el mayor número de los empleados administrativos (34, 33 y 23, respectivamente).

Por otro lado, los think tanks con el mayor número de investigadores contratados son los siguientes: ISEAS de Singapur, The Lowy Institute de Australia y VIE de Vietnam. Esto refleja su potencia investigadora dentro del sector y el potencial de producción científica de ellos.

Por último, cabe reflexionar sobre el tamaño de la estructura de estas organizaciones en la región investigada donde podemos ver que los think tanks vietnameses, singapurense, taiwaneses y australianos gozan de las estructuras de plantillas más grandes, siendo TIER el más grande de todos (360 empleados), marcando una diferencia muy significativa con las demás organizaciones. The Lowy Institute, ISEAS y VIE cuentan con una plantilla de entre 60 a 100 empleados, mientras ISET de Nepal y IDSS de Singapur son los think tanks más pequeños.

Anteriormente hemos destacado que el promedio del número de empleados es de 18 empleados, pero este gráfico nos permite observar que habitualmente las entidades emplean entre 20-40 personas, destacando este carácter organizacional de una pequeña o mediana organización (Figura 6). 


\section{Estructura de empleo}

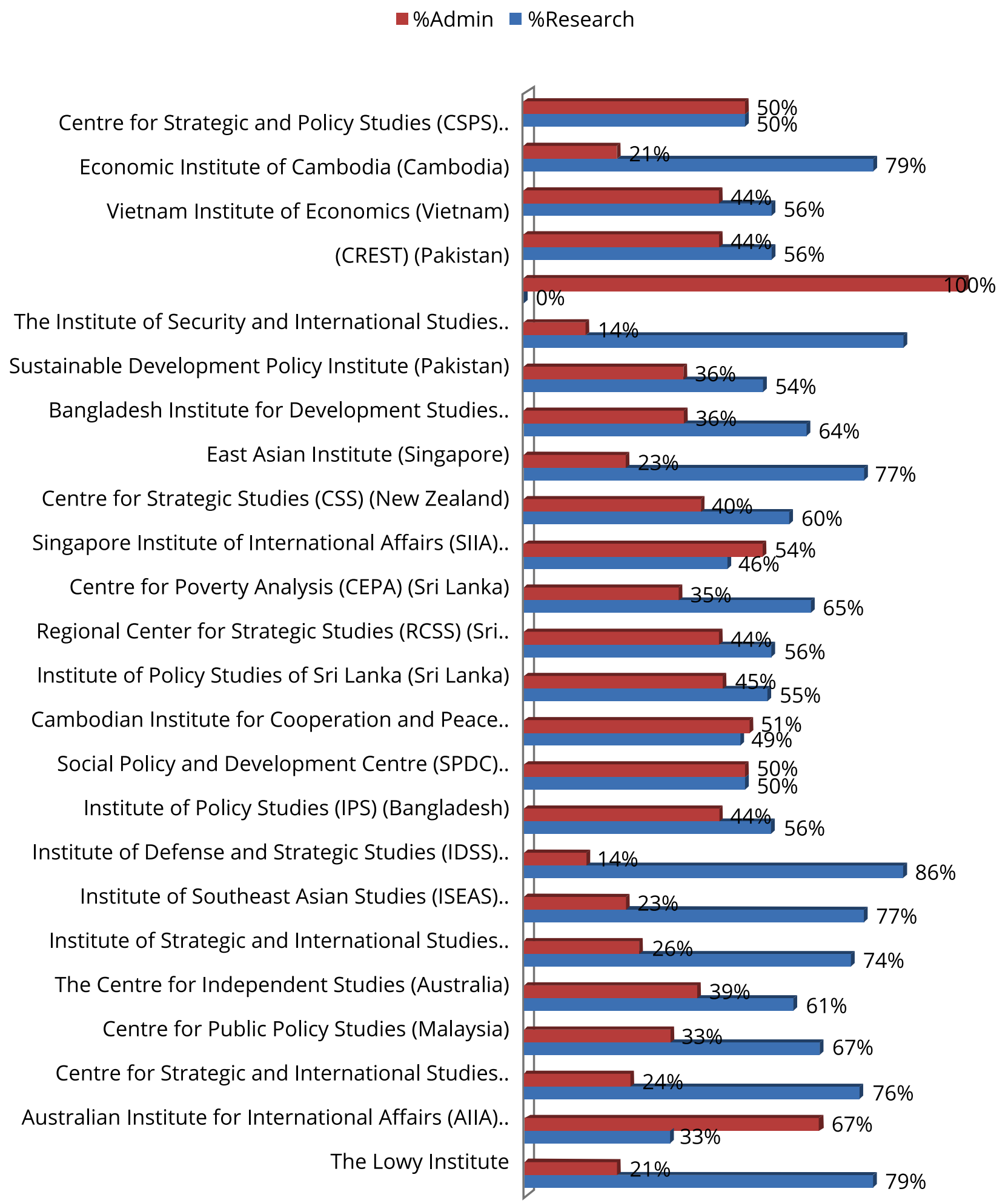

Figura 5. Estructura de empleo en los think tanks. Fuente: elaboración propia. 
Institute for Social and Enviromental Transition (Nepal) T 7 Institute of Defence and Strategicn Studies (IDSS) (Singapore) 7 Regional Center for Strategic Studies (RCSS) (Sri Lanka) -16 Institute of Policy Studies (IPS) (Bangladesh) $=16$ Centre for Strategic and Policy Studies (CSPS) (Brunei) -18 Taiwan Foundation for Democracy (Taiwan) $=20$ Centre for Strategic Studies (CSS) (New Zealand) -20 The Institute od Security and International Studies... 22 (CREST) (Pakistan) 23 Economic Institute of Cambodia (Cambodia) Social Policy and Development Centre (SPDC) (Pakistan) Centre for Public Policy Studies (Malaysia) Institute of Strategic and International Studies (ISIS) (Malaysia)

Australian Institute for International Affairs (AllA) (Australia)

Singapore Institute for International Affairs (SIIA) (Singapore) Centre for Strategic and International Studies (CSIS) (Indonesia) The Centre for Independent Studies (Australia) Sustainable Development Policy Institute (Pakistan) Foundation for Tolerance International (Kyrgyzstan) Centre for Poverty Analysis (CEPA) (Sri Lanka) East Asian Institute (Singapore) Institute of Policy Studies od Sri Lanka (Sri Lanka) Cambodian Institute for Cooperation and Peace (Cambodia) Bangladesh Institute for Development Studies (BIDS).. The Lowy Institute Vietnam Institute of Economist (Vietnam) Institute of Southeast Asian Studies (ISEAS) (Singapore) Taiwan Institute of Economic Research (TIER) (Taiwan)

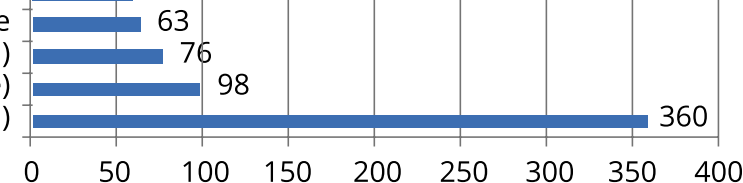

Figura 6. El tamaño de la estructura de los think tanks. Fuente: Elaboración propia.

Es importante señalar que existe un significativo número de los think tanks -14 (36\%) -que no publican de forma accesible y abierta la información sobre su estructura y tamaño de plantilla.

\section{$\%$ de los think tanks sin información sobre la plantilla}

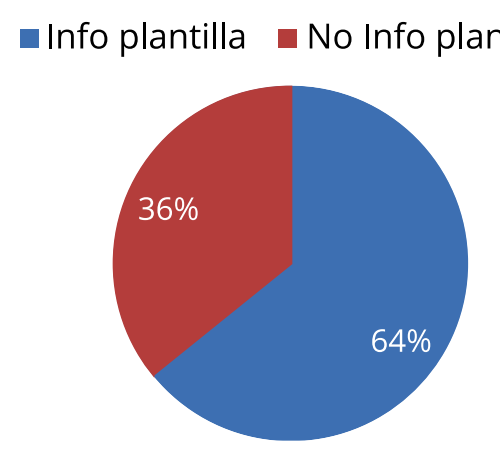

Figura 7. Los think tanks sin información en la web sobre la estructura y plantilla. Fuente: Elaboración propia.
Entre ellos son los siguientes:

- Political Risks Assessment Group (Singapore)

- Institute for Social and Environmental Transition (Nepal)

- Chung -Hua Institution for Economic Research (Taiwan)

- Taiwan Foundation for Democracy (Taiwan)

- Institute for Strategic and Development Studies (ISDS) (Philippines)

- Strategic and Defense Studies Centre (SDSC) (Australia)

- Economic Research Institute for ASEAN and East Asia (Indonesia)

- Taiwan Institute of Economic Research (TIER) (Taiwan)

- Center for Economic Research (Uzbekistan) 
- Institute of World Economics and Politics (IWEP) (Vietnam)

- Alternate Solutions Institute (Pakistan)

- Institute of National Capacity Studies (Indonesia)

- Darussalam Institute of Policy and Strategic Studies (BDIPSS) (Brunei)

- Diplomatic Academy of Vietnam (Vietnam)

El tamaño de la organización no tiene por qué influir sus funciones. Al investigar las informaciones de las organizaciones en sus páginas web, hemos podido concluir que sus funciones son similares entre ellas y muy típicas a los think tanks, tal como se recoge en la definición de este tipo de organización. Las funciones más destacadas son, tal como lo destaca en su página web The Lowy Institute: "to create ideas, research and advocacy, collaborations, communications, create a debate, education and training."

A pesar de la diversidad, hemos podido observar que, respecto a la procedencia y antigüedad, los think tanks son muy parecidos en cuanto se trata del tamaño de la organización, peso de investigación en el organigrama o sus funciones. También hemos podido observar que la gran mayoría de las entidades posee su propia página web. (Figura 8)

El 46\% de los think tanks declara en sus páginas web que son organizaciones no gubernamentales y sin ánimo de lucro. El segundo grupo más representado son los think tanks creados y gobernados por las entidades estatales, sobre todo en Vietnam y Taiwán, donde la relación entre estado y tercer sector es mucho más estrecha y ambigua. El 15\% son los institutos de investigación que pertenecen a las universidades o tienen estatus del centro de enseñanza. El 8\% se describe como empresas

\section{Tipo de organización}
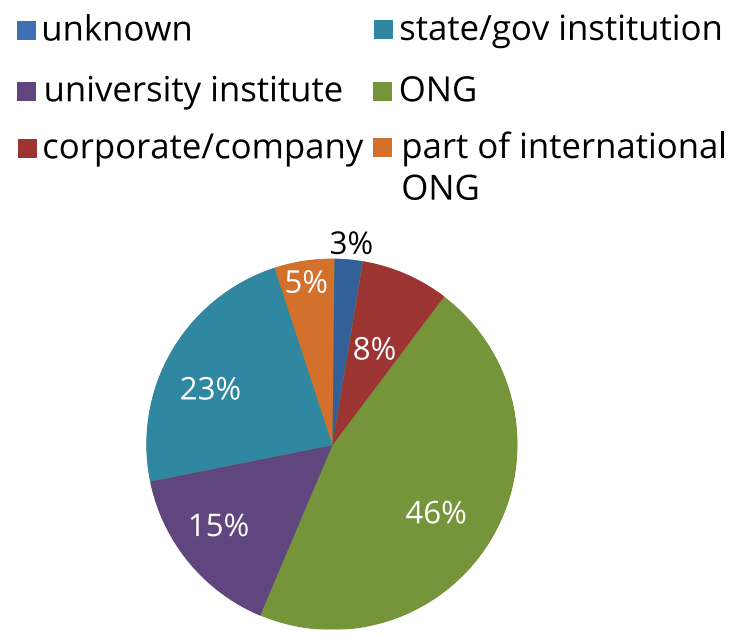

Figura 8. Tipología de los thinks tanks asiáticos. Fuente: elaboración propia

o corporaciones. El 5\% de ellos pertenecen a otras ONGs.

La gran parte presenta el modelo organizativo típico para los think tanks basado en el modelo de ONG, una organización sin ánimo de lucro, independiente del gobierno o como una fundación privada, al menos, en los principios de su creación. Como demuestra la Figura 10, 54\% de los think tanks asiáticos demuestra una diversidad en cuanto a su modelo de organización, cabe destacar que muchas de estas organizaciones en cuestión empezaron a constituirse como una ONG, pero desde una iniciativa gubernamental, como en caso de Pakistán o Bangladesh, para después operar como una entidad independiente.

El hecho de ser una ONG no significa de forma directa que el estatus de la organización es autónomo e independiente del gobierno. Especialmente, hemos observado que los think tanks de Vietnam y Taiwán tienden a declarar su modo de operación independiente siendo creados y gobernados por entidades del poder político y gobierno estatal. También, hay algunos think tanks, sobre todo ISIS Malaysia o 
Tailandia que pertenecen a las redes o asociaciones internacionales como ISEAN ${ }^{2}$ o en caso de FTI de Kirguistán de una ONG internacional (Figura 9).

En base a los datos arriba demostrados, podemos concluir que el $65 \%$ pertenece a las organizaciones totalmente independientes y el 77\% de ellas no pertenecen a las entidades globales, siendo los think tanks creados localmente.

\subsection{GRADO DE TRANSPARENCIA E INTERACTIVIDAD.}

Por último, hemos analizado el grado de transparencia de los think tanks asiáticos en cuanto a su financiación y fuente de ingresos, así como acceso a este tipo de información a través de la página web. Adicionalmente, de modo informativo, hemos recopilado la información sobre los dominantes modelos de financiación (Figura 10).

El 25\% de las organizaciones analizadas publica de forma clara y abierta sus fuentes de ingresos y modos de financiación, mientras que el 30\% no incluye ninguna información al respecto. El 45\% publica sólo la información sobre fondos de creación de la organización en sus principios, que suelen ser los fondos públicos (ministerios y gobierno, como en caso de Taiwán y Vietnam) o de algunas grandes ONGs internacionales (casos de Pakistán, Kirguistán o Uzbekistán).

También hemos observado financiación de las instituciones extrajeras (embajadas o las casas reales) y en el caso de los países desarrollados el el usual tipo de financiación del think tank es crear una fundación de fondos privados, trans-

2 El Anexo Número demuestra los think tanks que forman parte de ISEAN.

\section{Estatus}

$\square$ independent $\square$ dependent $\square$ no data

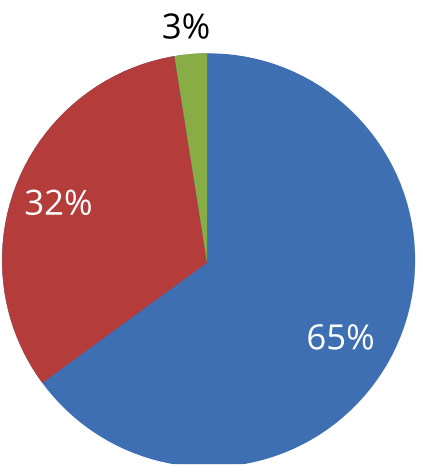

Figura 9. El estatus de los think tanks y pertenencia a las redes o asociaciones globales. Fuente: elaboración propia

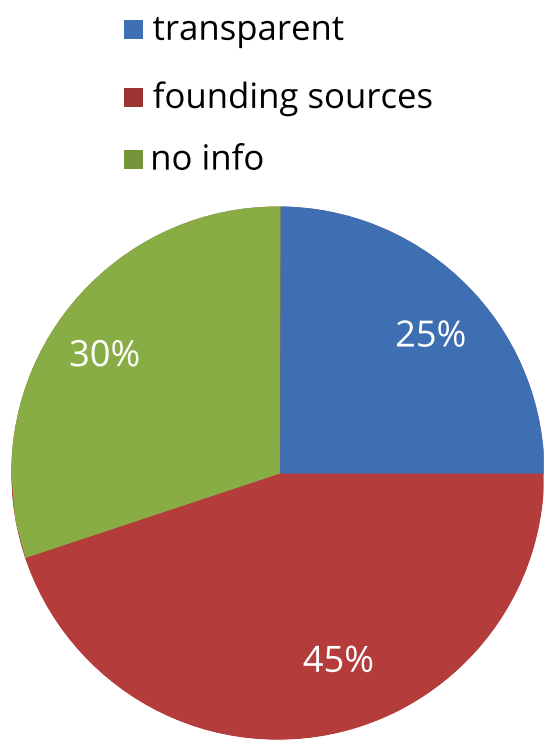

Figura 10. Grado de transparencia de la información sobre financiación. Fuente: elaboración propia.

formada después en una asociación. Este tipo de creación se observa en el caso de The Lowy Institute, SIIA, AllA, de Australia y Singapur en el caso de ISIS en Malaysia. De los 10 que declaran de forma abierta sólo 8 organizaciones indican de forma detallada todas sus fuentes 
de ingresos, mientras que en caso de AllA o The Lowy Institute podemos tener acceso a los informes financieros anuales y el detalle de los datos financieros, que fomenta la credibilidad de estas organizaciones.

Las fuentes de ingresos son muy diversas, las más populares son los siguientes: patrocinio, mecenazgos, subvenciones de las empresas privadas internacionales y nacionales, apoyo de fundaciones y ONGs internacionales, colaboraciones de investigación y relaciones multilaterales, royalties, publicaciones, entradas a los eventos, programas educativos y becas, donaciones, alquiler de propiedad, el propio fondo, membresía, contratación de investigación o servicios de consultoría al gobierno y otras entidades.

En general, los think tanks analizados muestran un nivel similar de interactividad a través de las redes de medios sociales, los sitios web y también en términos de adaptación a la nueva realidad 2.0, estando registrados en los niveles inferiores- por debajo y poco más de uno. Han adoptado herramientas, tácticas y estrategias similares respecto a las herramientas y redes sociales de forma similar.

Uso de herramientas interactivas en los sitios web corporativos se presenta en la Figura 11.

Como podemos observar, la conexión y la participación son las dimensiones más aplicadas, mientras que la presentación y el intercambio son las segundas. La colaboración y la revisión son aquellas con escasa aplicación entre estas organizaciones. En lo que respecta a los principales factores que determinan las dimensiones, los datos muestran que los centros de medios o las salas de redacción son el factor determinante para una presentación, el correo electrónico en el caso de la conexión, los botones de los Social Media para compartir y buscar en el caso de la participación. El factor de mayor importancia para determinar la dimensión de la colaboración es la adhesión. El nivel medio de interactividad se registra en el nivel medio-bajo, siendo la dimensión de la participación la más interactiva de todas ellas estando por encima de la media $(1,75)$, aunque la búsqueda como una herramienta más pasiva de participación es la más frecuente (Figura 12).

El nivel general de interactividad del contenido es de dos en una escala de tres grados, por lo tanto, es relativamente alto, aunque el tipo de contenido expositivo se utiliza con mayor frecuencia. La mayoría de los think tanks analizados utilizan un contenido rich media e interactivo en sus páginas web.

En términos de utilización de herramientas de medios sociales, en un primer momento hemos determinado las organizaciones con presencia en los Social Media. El cuadro general muestra que solo el $64 \%$ de los think tanks de Asia están presentes en los Social Media. La significativa mayoría de la muestra usa Facebook (96\%) y Twitter (76\%) y el 56\% de ellos tiene blog.

\section{CONCLUSIONES}

La página web se demuestra como una herramienta principal de relaciones públicas y muy bien establecida entre los think tanks en varios países, aunque la calidad de la misma puede variar y ser objeto de mejora. Es importante señalar que los think tanks investigados se dividen en dos grupos en lo referente a su antigüedad: los de desarrollo reciente y los think tanks creados en los años sesenta. Los think tanks fueron fundados principalmente por los gobiernos (los más antiguos), así como las 


\begin{tabular}{|c|c|c|c|c|c|}
\hline & $\mathbf{N}$ & $\%$ & VA & $\begin{array}{l}\text { Points } \\
\text { Awarded }\end{array}$ & $\begin{array}{l}\text { Medium In- } \\
\text { teractivity }\end{array}$ \\
\hline Presenting/Exposure & & & 1 & & \\
\hline Newsletter & 7 & $16 \%$ & & & \\
\hline Intranet & 2 & $4 \%$ & & & \\
\hline Library/Archive/edata/most read & 9 & $19 \%$ & & & \\
\hline Gallery & 2 & $4 \%$ & & & \\
\hline $\begin{array}{l}\text { networks/strategic alliances/international } \\
\text { cooperation/partners }\end{array}$ & 5 & $11 \%$ & & & \\
\hline Podcast & 4 & $9 \%$ & & & \\
\hline Webinars & 2 & $4 \%$ & & & \\
\hline Media Center/newsroom & 15 & $33 \%$ & & & \\
\hline \multirow[t]{2}{*}{ total: } & 46 & $100 \%$ & & 46 & 0,19 \\
\hline & $17 \%$ & & & & \\
\hline Connecting & & & 2 & & \\
\hline RSS & 10 & $13 \%$ & & & \\
\hline Register/account & 3 & $4 \%$ & & & \\
\hline Contact form & 18 & $23 \%$ & & & \\
\hline Email & 26 & $34 \%$ & & & \\
\hline Links to other centers & 9 & $12 \%$ & & & \\
\hline $\begin{array}{c}\text { Suscription to journals and other publica- } \\
\text { tions }\end{array}$ & 11 & $14 \%$ & & & \\
\hline \multirow[t]{2}{*}{ total: } & 77 & $100 \%$ & & 154 & 0,65 \\
\hline & $29 \%$ & & & & \\
\hline Sharing & & & 3 & & \\
\hline Social Buttons & 24 & $60 \%$ & & & \\
\hline Social Share & 12 & $30 \%$ & & & \\
\hline Recommend/add to Fav/tag & 4 & $10 \%$ & & & \\
\hline \multirow[t]{2}{*}{ total: } & 40 & $100 \%$ & & 120 & 0,51 \\
\hline & $15 \%$ & & & & \\
\hline Review & & & 4 & & \\
\hline Feedback/comment & 7 & $100 \%$ & & & \\
\hline \multirow[t]{2}{*}{ total: } & 7 & $100 \%$ & & 28 & 0,11 \\
\hline & $3 \%$ & & & & \\
\hline Participate & & & 5 & & \\
\hline Search & 32 & $39 \%$ & & & \\
\hline Download & 22 & $26 \%$ & & & \\
\hline
\end{tabular}




\begin{tabular}{|c|c|c|c|c|c|}
\hline Ecommerce/Products/Clients & 12 & $15 \%$ & & & \\
\hline workshps/trainings & 3 & $4 \%$ & & & \\
\hline other events & 10 & $12 \%$ & & & \\
\hline Contract research/Service/Projects & 3 & $4 \%$ & & & 1,75 \\
\hline total: & 82 & $100 \%$ & & 410 & \\
\hline Collaborate & $31 \%$ & & & & \\
\hline Collaboration as Expert/volunteer & 2 & $15 \%$ & & & \\
\hline Membership/Join & 6 & $47 \%$ & & & \\
\hline Donate /support & 5 & $38 \%$ & & & \\
\hline total: & 13 & $100 \%$ & & 78 & \\
\hline
\end{tabular}

Figura 11. Uso de herramientas interactivas en los sitios web corporativos. Fuente: Elaboración propia.

\begin{tabular}{|c|c|c|}
\hline Type of content on web & Formats/Freq Use \% & $\%$ of all TT \\
\hline \multirow[t]{2}{*}{ Expositive } & texts & $100 \%$ \\
\hline & Photos & $92.5 \%$ \\
\hline total & $24 \%$ & \\
\hline Medium Interactivity Level & 0,37 & \\
\hline \multirow[t]{2}{*}{ Hyper-textual } & Links & $92.5 \%$ \\
\hline & Hashtags & $33.3 \%$ \\
\hline total & $16 \%$ & \\
\hline Medium Interactivity Level & 0,51 & \\
\hline Participative & maps & $72 \%$ \\
\hline total & $9 \%$ & \\
\hline Medium Interactivity Level & 0,43 & \\
\hline Interactive & Videos/Audio & $85 \%$ \\
\hline \multirow[t]{2}{*}{ total } & $10.5 \%$ & \\
\hline & 0,67 & \\
\hline Immersive & Virtual visits & $0 \%$ \\
\hline total & $0 \%$ & \\
\hline Medium Interactivity Level & 0 & \\
\hline total Medium Interactivity & 2 & \\
\hline
\end{tabular}

Figura 12. La interactividad del contenido. Fuente: elaboración propia. 
organizaciones internacionales y los ONG (sobre todo los de reciente creación). Los temas principales a tratar son las relaciones y asuntos internacionales, desarrollo en general y la seguridad. Su ámbito principal de actuación y comunicación es la región de Asía y Pacifico. La mayoría de los think tanks investigados son las organizaciones del tamaño medio cuyas estructuras de empleo destaca el mayor peso de la investigación. En cuanto a su modelo organizativo destaca la diversidad y en ocasiones una transparencia limitada en lo referente al modelo final. Las organizaciones se presentan como independientes de las influencias de los gobiernos o partidos políticos con un enfoque claramente local. Los think tanks por norma general se caracterizan de una transparencia limitada en cuanto a la plantilla, estructura y sobre todo la financiación. Además, se puede observar un elevado nivel de la diversidad de las fuentes de financiación que utilizan los think tanks investigados. Por último, la página web ha demostrado ser una herramienta útil de transmitir la imagen deseada y fuente relevante de la información sobre la organización. Como tal, sirve como la primera plataforma de contacto en el mundo 2.0 y de este modo ayuda a iniciar las relaciones con los públicos.

El estudio confirma que las páginas web, como una herramienta de comunicación y canal de información corporativa, resulta útil para recopilar los datos básicos y generales de la organización en el $97 \%$ de los casos, (teniendo en cuenta que sólo un think tank no tuve la página web).

Se observa un estable y continuo crecimiento de este tipo de organizaciones en la región en cuestión con unas épocas claramente marca- das por un boom en la creación de ellas, reflejando de esta forma los procesos democráticos y políticos internacionales y locales. El reciente aumento del número de los think tanks establecidos destaca el incremento de la importancia de ellos en la arena política en países de Asia y Pacifico. Su creación se debe a los factores y la iniciativa locales, ya sea inicialmente por el gobierno o alguna ONG internacional, ya que la mayoría de ellos no forma parte de los think tanks globales o redes globales. Aunque hay que admitir, que en muchas ocasiones este tipo de información no está mostrada de forma muy clara y exacta en la página corporativa.

Esta actividad abarca las funciones que los estudios de think tanks enmarcan en su definición, funciones que todas las organizaciones que hemos estudiado comparten de forma general: investigación y su difusión, creación de ideas y espacios para el debate, tareas relacionadas con la educación y enseñanza, así como la advocacy (defensa). Estas funciones las llevan a cabo las organizaciones de un tamaño medio de 18 empleados, con el tamaño dominante de la estructura de entre 20 y 40 personas, siendo en su mayoría las organizaciones medianas o más bien pequeñas, ya que el estudio ha revelado que sólo 4 think tanks cuentan con más de 60 y casi un centenar de empleados y sólo uno con 360 empleados.

La dedicación a la investigación como la actividad principal y básica de este tipo de organizaciones en la región en cuestión, se refleja en la estructura de los empleados, en la cual prevalecen los empleados dedicados a las tareas de investigación. Cabe destacar que existe un número considerable de los think tanks asiáticos que no demuestran de forma clara la es- 
tructura y tamaño de su plantilla lo que pueda influir la percepción acerca de su transparencia y credibilidad de forma parecida a la situación anteriormente mencionada en referencia a la pertinencia o no a las organizaciones globales. Los think tanks más relacionados con las iniciativas de gobiernos suelen tener más empleados administrativos, mientras que las entidades universitarias, el personal investigador (AllA con $67 \%$ de administración, SIIA con 54\% o CICP con 51\%), mientras que las entidades universitarias, el personal investigador (más de 60\%, como por ejemplo EIC, CEPA, CIS, etc.).

Los datos sobre los tipos de organización reflejan la mayor diversidad entre los think tanks investigados lo que confirma una tendencia general dentro del sector donde, por un lado, el modelo dominante son ONGs (sin ánimo de lucro y no gubernamentales) y por otro, se puede observar un grado elevado de diferenciación del tipo de organización bajo la cual funcionan. En este estudio, la mayoría opera bajo los modelos distintos a las ONGs declarados por un 46\%, siendo también en un número significativo los tipos de organizaciones como institutos de investigación de alguna universidad o de ministerio o gobierno, así como de una u otra ONG.

Dicha diversidad de modelos de organización queda reflejada también en los modelos de financiación y las fuentes de ingresos de los think tanks analizados. En cuanto a los principios de su fundación, hemos observado que dominan los fondos gubernamentales, de las ONGs internacionales y los privados. Incluso, un considerable número de los think tanks en los países con más dificultades políticas y económicas como por ejemplo Pakistán, se ha creado con fondos estatales o internacionales para después alcanzar la independencia en su financiación y funcionamiento.

Los modelos de financiación más indicados son subvenciones, donaciones, colaboraciones, venta de las publicaciones o entradas. En este punto es de mayor importancia destacar que sólo un 25\% pública la información detallada sobre sus fuentes de ingresos e incluso menos think tanks publican de forma abierta sus informes financieros. Un segmento amplio da acceso sólo a la información acerca de los fondos iniciales del momento de su creación y un 30\% no publica ninguna información al respecto. Estos datos revelan las cuestiones relacionadas con la transparencia y credibilidad que deberían ser abordadas por la mayoría de los think tanks asiáticos de forma adecuada. Si añadimos a esto falta de información sobre las plantillas de los empleados y relaciones con redes o ONGs globales, parece que la cuestión de información abierta y pública, accesible a través de la página web debería formar una prioridad comunicativa de estas organizaciones en la región asiática, sobre todo en la era digital y comunicación 2.0 en la cual transparencia, credibilidad y una información exacta goza de una importancia aún mayor a la hora de establecer las relaciones con los stakeholders a través de la actividad comunicativa.

Por último, el análisis ha revelado que los think tanks han apostado por una relativa interactividad en sus espacios web, aunque el mayor peso reside en la interacción enfocada en el contacto y participación en las publicaciones de la organización en su página web y en la exposición del contenido audiovisual. 


\section{DISCUSIÓN.}

La capacidad de los think tanks para generar espacios comunicativos se puede concretar en numerosas herramientas de comunicación, cada una de ellas con sus ventajas y sus inconvenientes. En el caso de los espacios web nos permiten mantener un canal fluido y permanente de comunicación con otros actores comunicativos como son los medios de comunicación y con nuestros públicos. A través de la web, los think tanks son capaces de comunicarse de manera directa, pero también debemos ser conscientes que existen otras posibilidades como las redes sociales o la presencia en medios de comunicación.
En este sentido, las páginas web son una herramienta común y suficientemente bien desarrollada en las estrategias de las relaciones públicas políticas 2.0 de los think tanks ubicados en Asía, por lo cual afrontaron con éxito el reto digital (McGann, 2015).No obstante, no están libres de las cuestiones relacionadas con la transparencia sobre todo en cuanto a temas financieros y administrativos (plantillas, etc.) y como tal pueda afectar el modo de establecer y desarrollar las relaciones con los stakeholders (Wilox et al., 2012). Sin embargo, las páginas web como las principales tarjetas que transporten la imagen de estas organizaciones en el mundo digital hacía sus públicos deberían ser sujetas a las mejoras en el futuro próximo con el fin de adaptarse mejor a la dinámica del mundo comunicativo denominado 2.0 (Liberos, et al., 2013), sobre todo en cuanto se trata del nivel de transparencia e interactividad (Aced, 2013; Capriotti, Carretón y CastiIlo-Esparcia, 2016) de las mismas con el fin de fomentar las relaciones con los públicos. 


\section{BIBLIOGRAFÍA}

Abelson D. (2001). Do The Think Tanks Matter? Asessing The Impact of Public Policy Intitutes. Montreal: McGill-Queen's University Press.

Abelson, D. (2006). A Capitol Idea. Think Tanks and US Foreing Policy. Montreal: McGill-Queen's University Press.

Aced, C., Arqués, N., Benítez, M., Llordá B., y Sanagustin E. (2009). Visibilidad: cómo gestionar la reputación en Internet. Barcelona: Ediciones Gestión 2000.

Aced C. (2013). Relaciones Públicas 2.0. Barcelona: Editorial UOC.

Barquero Cabrero, J.D., Peréz Senac R., y Barquero Cabrero M. (2010). Dirección estratégica de relaciones públicas. Barcelona: Profit Editorial.

Capriotti P., Carretón, M.C. y Castillo-Esparcia, A. (2016). Testing The Level of Interactivity of Institutional Websites: From Museum 1.0 To Muesums 2.0. International Journal of Information Management, 36, 97-104

Duhé, S. (2015). An Overview of New Media Research in Public Relations Journals from 1981 To 2014. Public Relations Review, 41, 153-169.

Hall, P. (1990). Policy Paradigms, Experts and the State: The Case of Macroeconomic Policymaking in Britain. En S. Brooks, y A.G. Gagnon, (ed.), Social Scientists, Policy and the State. Greenwood, NJ: Praeger Publishing House.

Holtz, S. (2002). Public relations on the Net: winning strategies to inform and influence the media, the investment community, the government, the public, and more (2nd ed.). New York: Amacom

Kent, M. L., \& Taylor, M. (1998). Building Dialogic Relationships Through the Worldwide Web. Public Relations Review, 24(3), 321-334.

Kent, M. L., Taylor, M., \& White, W. J. (2003). The relationship between Web site design and organizational responsiveness to stakeholders. Public Relations Review, 29(1), 63-77.

Krastev, I. (1999). The Liberal Estate. East Central Europe 26/1.

Krastev, I. (2001). Think tanks: Making and faking influence. Southeast European and Black Sea Studies, 1(2), 17-38.

Liberos, E. (coord.) (2013). El libro de Marketing Interactivo y la Publicidad Digital. Madrid: ESIC Editorial.

McGann, J., Weaver R. K. (eds.) (2000). Think Tanks and Civil Societies: Catalysts for Ideas and Action. Sommerset, NJ: Transaction Press.

McGann, J. (2007). The Global "Go-to Think Tanks. The Leading Public Policy Research Organizations in the World. Philadelphia: Foreign Policy Research Institute.

McGann, J.G. (2013). 2013 Global Go to Think Tanks Index Report. Philadelphia: University of Pennsy/vania. Fuente: http://gotothinktank.com/dev1/wpcontent/uploads/2014/01/GoToReport2013.pdf 
McGann, J.G. (2015). 2014 Global Go To Think Tanks Index Report. Philadelphia: University of Pennsy/vania. Fuente: http://repository.upenn.edu/think _tanks $/ 8$

Rich, A. (2004). Think Tanks, public politics and the politics of expertise. Cambridge: Cambridge University Press.

Rojas Orduña O.I. (2012). Relaciones Públicas: La eficacia de la influencia. ESIC Editorial, Madrid

Seitel, F. P. (2002). Teoría y Práctica de las Relaciones Públicas 2.0. Madrid: Pearson Prentice Hall.

Selee, A. (2013). What Should Think Tanks Do? A Strategic Guide to Policy Impact. Stanford: Stanford Briefs.

Smith, J. A. (1991). The Idea Brokers: Think Tanks and the Rise of the New Policy Elite. New York: The Free Press.

Slevin, J. (2009). The Internet and Society. Cambridge, MA, USA: Balckwell Publishers

Solis, B., Breakenridge D. (2010): PR 2.0 in a Web 2.0 world: what is public relations 2.0. New Jersey: FTPress Delivers.

Stone, D. (2005). Think Tanks and Policy Advice in Countries in Transition, Marie Curie Chair \& Head, Public Policy Program Central European University. Paper prepared for the Asian Development Bank Institute Symposium: "How to Strengthen Policy-Oriented Research and Training in Viet Nam". 31st August 2005, Hanoi, Vietnam.

Valentini, C. (2015). Is Using Social Media Good for The Public Relations Profession? A Critical Reflection, Public Relations Review, 41, 170-177.

Van DijkJ. (1991). The Network Society: Social Aspects of New Media. Houten: Bohn Staflen Van Loghum.

Van Ruler, B. (2015). Agile Public Relations Planning: The Reflective Communication Scrum, Public Relations Review, 41, 187-194.

Weaver, K. (1989). The Changing World of Think Tanks. Political Science and Politics, Vol.22 (3), 563-578.

Verčič, D., Verčič Tkalac A., y Sriramesh K. (2015). Looking for digital in public relations. Public Relations Review, 41, 142-152.

Wilcox, D.L., Cameron G.T., Xifra J. (2012). Relaciones Públicas. Estrategias y tácticas (10ª Edición). Madrid: Pearson.

Xifra, J. (2008). Los think tanks. Barcelona: Editorial UOC.

Zerfass, A., Tench, R., Vercic, D., Verhoeven, P., y Moreno, A., (2014). European Communication Monitor 2014. Excellence in strategic communication Key issues, leadership, gender and mobile media. Results of a survey in 42 countries. Brussels: EACD/EUPRERA, Helios Media. Recuperado de: http://www.zerfass.de/ECM-WEBSITE/media/ECM2014-Results-ChartVersion.pdf. 